\title{
Position du film traduit en Europe et en Pologne : 27 ans après
}

\section{Abstract \\ Position of Translated Film in Europe and in Poland: 27 Years Late}

The position of Polish film after 1989 has significantly deteriorated. Previously admired and respected, Polish cinematographers are now battling against a profound crisis - a creative one, but also a financial one.

In the meantime, American films, i.e. Hollywood films, dominate in European cinemas. And as, according to Lawrence Venuti, a translation strategy concerns also the choice of texts to be translated, this is the point in which translation commences. Therefore, why do the majority of films selected for translation by European distributors come from Hollywood?

Since the 1980s, a period of extraordinary prosperity for Hollywood, and a period of crisis for the European cinema, the manner of perceiving of motion pictures has changed, and that, in turn, has changed the balance of power in the world cinema. We shall briefly present those changes and factors that influenced them, and shaped the current situation. In order to better illustrate it, we shall base the presentation on Zohar's polysystem theory.

This situation influences the position of Polish cinema: recognized and appreciated for its artistic value in the period between 1950s and 1980s, despite censorship, and now - a marginal phenomenon, which may seem surprising, even paradoxical. We shall attempt to demonstrate also possible explanations for that situation.

At the end of our paper, we shall briefly comment on the status of the television, which is slightly different than that of the cinema.

Keywords: Polish film, Hollywood film, audiovisual translation market, cinema, television.

\section{Comme l'a dit Juliusz Machulski pendant le débat sur le cinéma polonais :}

A l'époque de la Pologne communiste, nous étions capables de rivaliser avec le monde, même dans le domaine du film de divertissement, parce que l'argent était artificiel. Les films coûtaient autant qu'ils devaient coûter, il y avait autant de jours de tournage qu'il était nécessaire. [...] Je ne veux pas dire que c'était grâce au communisme ; je veux dire 
que c'était bon car il y avait des ressources. Nous nous sentons investis d'une mission, et cependant nous oublions une chose prosaïque : l'argent ${ }^{1}$.

La situation du film polonais après 1989 s'est considérablement détérioré. Autrefois admirés et respectés, les cinéastes polonais se battent aujourd'hui contre une crise profonde - une crise créative, mais aussi une crise financière.

Cependant, dans les cinémas européens prédomine le film américain, c'est-à-dire le film hollywoodien. Et comme, d'après Lawrence Venuti, la stratégie de traduire concerne aussi le choix des textes à traduire ${ }^{2}$, la traduction commence également en ce moment. Pourquoi donc la majorité de films choisis pour la traduction par les distributeurs européens vient d'Hollywood?

Depuis les années 80., étant une période d'une prospérité extraordinaire pour Hollywood, et une étape de crise pour le cinéma européen, a changé la manière de percevoir l'œuvre cinématographique, ce qui a déterminé les rapports de force dans le cinéma mondial. Nous allons présenter brièvement ce changement, les facteurs qui l'ont influencé et qui ont façonné la situation courante. Pour mieux l'illustrer, nous allons fonder notre présentation sur la théorie « revisée » des polysystèmes d'Itamar Even-Zohar.

Cette situation influence la position du cinéma polonais: reconnu et apprécié pour sa valeur artistique pendant les années 1950 et jusqu'à 1980, malgré la censure, tandis que maintenant il est plutôt un phénomène marginal, ce qui peut sembler surprenant, voire paradoxal. Nous allons tenter de démontrer également l'explication possible de cette situation.

A la fin de notre exposé, nous allons également commenter brièvement le statut de la télévision, qui est un peu différent de celui du cinéma.

\section{Le marché cinématographique polonais et mondial}

Voici le classement des meilleurs films de 2015 d'après la critique polonaise (les films polonais mis en gras) :

1. Whiplash

2. Foxcatcher

3. The Imitation Game

\section{Wkręceni 2}

5. Birdman

6. The Theory of Everything

7. Penguins of Madagascar

8. Ziarno prawdy

9. Fifty Shades of Grey

10. American Sniper

1 J. Machulski, Polskie filmy to najczęściej nuda: debata o kinie, apud M. Adamczak, Globalne Hollywood, filmowa Europa i polskie kino po 1989 roku, Gdańsk 2010, p. 233, traduction nous-même.

${ }^{2}$ L. Venuti, Translation Strategy [dans :] idem, Routlege Encyclopaedia of Translation Studies, Londres 2000, p. 240-241.

Voir aussi: L. Venuti, The Scandals of Translation: Towards an Ethics of Difference, London 1998. 


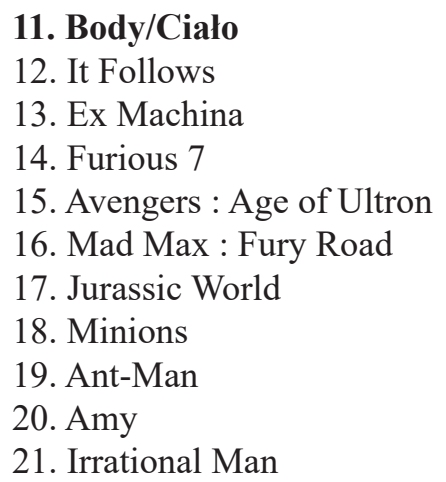

22. Mission: Impossible 5

\section{Karbala}

24. Youth

25. The Martian

26. Spectre

27. Bridge of Spies

28. Steve Jobs

29. Hunger Games : Mockingjay Part 2

30. Star Wars Episode VII : The Force Awakens

Ce classement a été préparé par les critiques de cinéma collaborant avec le site stopklatka.pl, consacré à la cinématographie mondiale. Comme nous le voyons, il n'y a que quatre films polonais, tandis que 26 d'entre les meilleurs films de l'année 2015 sont des films américains. Cette tendance dominatrice est caractéristique pour la plupart de classements et statistiques polonais, européennes et mondiales, qui ont été resumées dans le diagramme 1 créé par Marcin Adamczak.

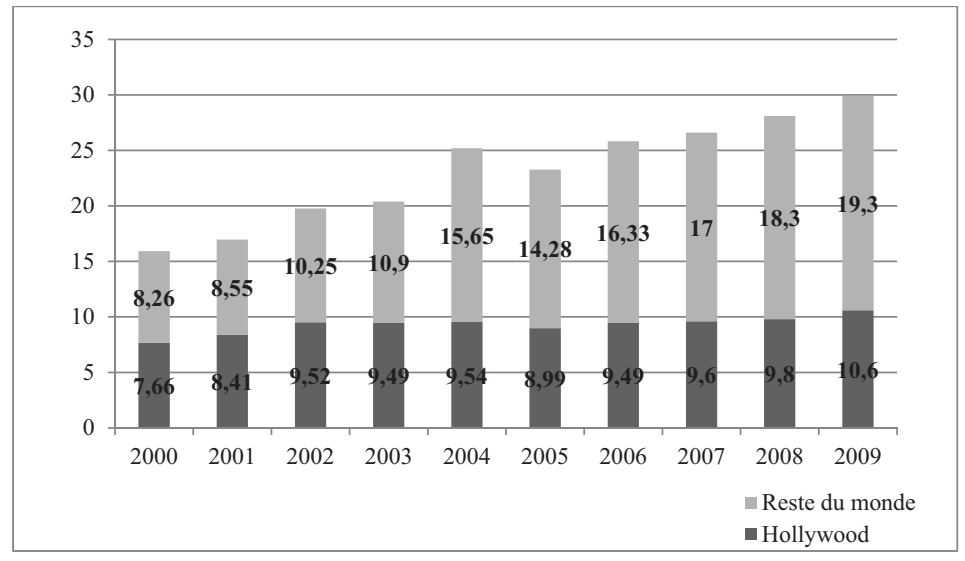

Diagramme 1. Cinématographie mondiale contre la cinématographie hollywoodienne. Source: M. Adamczak, Globalne Hollywood, filmowa Europa i polskie kino po 1989 roku, Gdańsk 2010, p. 448. 
Ce diagramme démontre la participation de l'Hollywood dans le marché cinématographique mondial (profits en millions de dollars). Evidemment, la cinématographie hollywoodienne constitue au moins un tiers de toute l'industrie du cinéma mondial - en d'autres termes, on peut dire qu'Hollywood occupe une position centrale dans le polysystème cinématographique - non seulement à l'échelle mondiale, mais dans la majorité de pays européens, y compris la Pologne ! Il semble que nous ne voulions plus voir de films faits chez nous, et qui plus est, que nos réalisateurs les plus doués et reconnus ne veuillent pas tourner de films dans notre pays. Pourquoi?

\section{Polysystème du cinéma : facteurs déterminant l'organisation des éléments}

Itamar Even-Zohar, dans son texte « canonique » du 1979, mentionne trois facteurs qui déterminent l'organisation du centre et des périphéries dans un polysystème (il parle du polysystème littéraire, mais dans son texte revisé, il élargit cette catégorie sur toute la culture) :

1. lorsqu'une littérature/culture est « jeune »- en cours de se former ;

2. lorsqu'une littérature/culture est « faible »-quelques éléments manquent dans son répertoire ;

3. lorsqu'il y a un « vide » créatif, une littérature/culture se trouve en état de crise. $^{3}$

Il est évident qu'en ce qui concerne le polysystème cinématographique, cette liste - celle d'origine et sa version modifiée - manque d'un aspect très important : l'aspect financier. Il est important surtout pour le cinéma d'aujourd'hui, et en particulier pour le cinéma américain.

Depuis les années 1980, Hollywood a évolué très rapidement. La méthode de financement de productions cinématographiques a changé : les anciens «barons » ont été remplacés par des managers en colliers blancs ; c'est-à-dire, les corporations ont remplacé les studios gérés par les cinéastes : les studios du Big Six (les studios hollywoodiens les plus grands) ont été associés aux grandes multinationales (p.ex. Columbia Pictures fait partie du groupe Sony), médias, maisons d'édition, etc. Par conséquent, le film est devenu produit; en outre, il est devenu « un produit qui sert à vendre d'autres produits ${ }^{4}$ ». Il ne s'agit plus de l'art, mais du business. Il ne s'agit plus de comment tourner un film, mais de comment le vendre, et de voir ce qui peut être vendu avec celui-ci ${ }^{5}$. Pour mieux contrôler le marché, les Big Six utilisent des stratégies du marketing, telles que blanket strategy (« stratégie du tapis de bombes ») et la multiplexisation : cela consiste

3 I. Even-Zohar, Miejsce literatury tlumaczonej w polisystemie literackim [dans :] Wspótczesne teorie przekładu. Antologia, éds. P. Bukowski, M. Heydel, Kraków 2009, p. 199; les théories présentés dans cette publication ont été revisées et développées dans: Polysystem Theory (Revised) [dans :] I. Even-Zohar, Papers in Culture Research,Tel Aviv 2010, p. 40-51.

4 M. Adamczak, op. cit., p. 158 (traduction nous-même).

5 R. Maltby, Commercial Aesthetic [dans :] idem, Hollywood Cinema. An Introduction, Oxford 1995 , p. 7.

Voir aussi : Kino klasyczne, éds. T. Lubelski, I. Sowińska, R. Syska, Kraków 2011. 
en création de grands réseaux de cinémas, associés à lesdits studios, et lancement des nouveaux films dans le réseau entier, simultanément. Il est donc évident que cette stratégie vise à atteindre le plus grand public possible. L'effort financier est donc énorme (le budget moyen d'une production américaine se situe entre 70 et 100 millions de dollars par film !) et une bonne partie du budget est dépensée en publicité. Les grands studios ne peuvent pas se permettre d'échec : chaque film doit générer des profits, non seulement au box office, mais aussi quant aux émissions consécutives dans la télévision, vente des disques DVD et Blu-Ray, jeux vidéo et d'autres produits secondaires, créés pour atteindre cette partie du public qui ne va pas aux cinémas.

En Europe, les cinéastes perçoivent leurs productions d'une manière différente : le film reste toujours et avant tout une œuvre d'art, il fait donc partie du patrimoine national. C'est le rôle de l'auteur, et non des vendeurs, qui est décisif. L'aspect commercial, certes non negligeable, reste moins important ; le public cible est la critique et les participants des festivals. Les productions sont financées par les organisations et les institutions (comme PISF en Pologne), dans la plupart des cas, cofinancées par l'Etat. L'effort financier est beaucoup moins élévé qu'aux Etats-Unis (p.ex. budget moyen d'un film anglais : 13 millions \$, d'un film français : 7 millions \$) et la publicité est limitée. Le nombre de films produits par an n'a pas autant d'importance : aux Etats-Unis, chaque année 350 films sont lancés dans les salles de cinéma, mais, par exemple, en France en 2013 il y en avait $258^{6}$ !

\section{Position du cinéma polonais avant et après 1989}

La situation en Pologne est encore plus compliquée : avec la chute du communisme a changé aussi le système du financement des productions cinématographiques, mais les réformes ont été introduites trop lentement et d'une manière incohérente. Avant 1989, tous les films ont été financés par l'état; maintenant, le budget des productions cinématographiques polonaises est un " patchwork » de ressources fournies par l'état, les agences, fondations, corporations médiatiques (tel ITI) et investisseurs privés. Et pourtant, il est toujours réduit par rapport à l'époque communiste : le budget moyen des films polonais se situe entre 2,5 et 3 millions de dollars (notre « super-production », le film le plus coûteux de l'histoire du cinéma polonais - Quo Vadis - a coûté 68 millions zlotys, ce qui constitue toujours un montant minime par rapport à des blockbusters hollywoodiens) ; avant 1989, le financement était relativement plus élévé, et de plus, beaucoup de processus pendant le tournage ont été effectués « hors paiement» : par exemple, les scènes de bataille ont été réalisées avec la participation de l'armée polonaise? Qui plus est, la loi communiste imposait que la majorité de films en projection dans les cinémas polonais viennent du bloc de l'Est (78\% dans les années 1960,

6 Statistiques du centre national du cinéma et l'image animée, www.cnc.fr/web/en/statistics (consulté le 20.11.2014).

7 M. Adamczak, op. cit., p. 234. 
54\% dans les années 1980), ce qui favorisait les œuvres des cinéastes polonais, et c'étaient les cinéastes qui décidaient quels projets devaient être choisis et réalisés avec les ressources de l'état. C'est peut-être la raison pour laquelle « l'école cinématographique polonaise » était à l'époque considérée l'un des courants artistiques européens appréciés (malgré la censure !) ; et c'est pourquoi Tadeusz Lubelski décrit le système communiste (dans le contexte du cinéma, bien entendu) comme « autant inconvénient qu'avantageux ${ }^{8} »$.

Pourquoi le budget est-il tellement important? Tout d'abord, il se traduit non seulement en toute «l'infrastructure » comme les décors, les costumes, etc., mais aussi en jours de tournage et de post-production : plus on a de ressources, plus soigneusement on peut réaliser un projet cinématographique. Dès lors, comme les projets polonais ne sont pas suffisamment financés, les productions sont moins élaborées. Les cinéastes appréciés (tels Roman Polański, Agnieszka Holland) créent en majorité à l'étranger ou profitent des financements européens, et en conséquence, peu de films en langue polonaise sont reconnus dans le monde.

\section{La télévision : un cas à part}

La seule entité à s'opposer à la suprématie d'Hollywood est la télévision - tant en Pologne que dans les autres pays européens. Comme nous l'avons déjà mentionné, la télévision aux Etats-Unis sert à transférer les films de la « Fabrique des Rêves » à un public encore plus grand ; cependant, les proportions entre le contenu étranger et le contenu national dans la télévision en Pologne (et en Europe en général) sont beaucoup plus équilibrées qu'au cinéma. Cette situation est due (entre autres facteurs) à une réforme et une privatisation beaucoup plus rapides et mieux organisées par rapport à la réorganisation du système cinématographique.

Dans la table 1 nous présentons ci-dessous une brève comparaison entre la structure de la télévision avant 1989 et aujourd'hui'.

Table 1. Chaînes télévisées en 1989 et aujourd'hui

\begin{tabular}{|l|l|}
\hline Avant $\mathbf{1 9 8 9}$ & $\mathbf{2 0 1 6}$ \\
\hline 3 chaînes (TVP1, TVP2, Polonia) & Presque $\mathbf{2 0 0}$ chaînes en langue polonaise \\
\hline Financement: état & Financement: état/corporations \\
\hline $\begin{array}{l}\text { Programme: majorité de films et séries TV polo- } \\
\text { nais; quelques télénovelas de l'Amérique du Sud } \\
\text { et films étrangers; plusieurs films du bloc de l'Est; } \\
\text { émissions documentaires et émissions de divertis- } \\
\text { sement - polonaises }\end{array}$ & $\begin{array}{l}\text { Programme: majorité de films hollywoodiens, } \\
\text { mais presque autant de séries TV polonaises; } \\
\text { sement - polonaises et étrangères }\end{array}$ \\
\hline
\end{tabular}

8 T. Lubelski, Film fabularny [dans :] idem, Encyklopedia kultury polskiej XX wieku. Film. Kinematografia, p. 173.

9 Ce sommaire a été préparé sur la base d'une analyse minutieuse des programmes de chaînes TV d'avant 1989 et 2016. Source des données: stopklatka.pl (consulté le 20.11.2014). 
Comme nous pouvons le constater, l'Hollywood est toujours présent dans la télévision polonaise d'aujourd'hui, mais le contenu (du moins dans les chaînes privées les plus répandues, tels TVN et Polsat) - ce qui peut surprendre - est beaucoup moins « américanisé » que dans le cas du cinéma. Par ailleurs, d'après les chercheurs de Film Studies, cette tendance est caractéristique pour toute l'Europe $^{10}$.

Bien sûr, parmi ces 200 chaînes, il y a plusieurs (et de plus en plus) programmes qui sont lancés par des groupes médiatiques étrangers, comme toutes les « variations » de la BBC, MTV ou HBO : la plupart (ou presque l'ensemble) d'émissions diffusées dans ces chaînes sont des programmes documentaires, films et émissions de divertissement en anglais; ils doivent donc être traduits pour la diffusion en Pologne.

En ce qui concerne les conséquences de cette situation pour le marché de la traduction audiovisuelle, la quantité d'émissions, séries, films et programmes à traduire a augmenté énormément ; mais puisque le contenu est très varié, et les traducteurs audiovisuels ne sont toujours pas assez nombreux, la qualité de plusieurs de ces traductions, surtout dans la télévision, et surtout pour les programmes pas trop populaires, hors prime time, est douteuse. Ce problème concerne moins le cinéma, parce que c'est aux meilleurs traducteurs que sont donnés la plupart de films à traduire.

En résumé, depuis les années 1980 le cinéma hollywoodien évoluait; au XXI siècle, il est devenu une industrie très complèxe (et efficace) qui crée des produits de consommation : évidemment, cette stratégie n'est pas très ambitieuse du point de vue artistique, mais elle est certainement profitable : Hollywood a dominé la cinématographie mondiale, tandis que le cinéma européen - « cinéma de création », géré et perçu différémment, reste un phénomène « niche ».

En ce qui concerne le cinéma polonais, on peut dire qu'après la chute du communisme une cinématographie autrefois admirée est devenue « périphérique à la périphérie » du polysystème, pour plusieurs raisons ; parmi les facteurs déterminant cette situation les plus importants semblent l'effort financier faible, ainsi que pas assez de dépenses pour les activités publicitaires.

Le seul domaine qui a réussi à éviter l'hégémonie de la Fabrique des Rêves est, comme nous avons souligné, la télévision : grâce à une réforme rapide et efficace, les chaînes les plus répandues ont plusieurs productions en polonais : des séries télévisées, émissions de divertissement, des « formats » adaptés aux conditions polonaises, etc.

Quant aux « rapports de force » à l'intérieur du polysystème, la position marginale du nouveau cinéma polonais n’est pas liée aux phénomènes « types » mentionnés par Zohar : le cinéma européen, et surtout le cinéma polonais, peut être considéré comme «jeune », mais seulement en ce sens qu’il n’a pas assez d'expé-

10 Depuis quelques mois, avec le lancement des plate-formes comme Netflix et YouTube Red en Europe, et la croissance soudaine de la popularité des séries télévisées, cette tendance devient de plus en plus visible: il y a, bien sûr, des séries américaines très répandues, mais les séries vraiment « cultes » viennent le plus souvent du Royaume-Uni (p.ex. Sherlock ou Utopia); il y a aussi des séries scandinaves très ambitieuses (comme Trapped - série islandaise, ou Real Humans - série suédoise). 
rience de l'utilisation des outils modernes : technologie de pointe, méthodes de promotion, etc. ; de plus, le manque d'inspiration, d'artistes doués, ne concerne pas le cinéma polonais - l'abolition de la censure et la possibilité d'échange d'idées entre la Pologne et l'Ouest a plutôt élargi les horizons des cinéastes, leur donnant des possibilités nouvelles d'expression; on peut donc constater que dans le cas du cinéma, le côté économique est d'une influence décisive.

Et finalement, qu'est-ce que cela signifie pour le marché de traduction audiovisuelle ? Du point de vue des traducteurs et des traductologues, cette situation est sans doute avantageuse. Il y a beaucoup de matériel à traduire et à analyser : séries télévisées, émissions documentaires et de divertissement, et surtout les films - créés pour la projection cinématographique ou bien pour la diffusion à la télévision, parfois nécessitant des traductions nouvelles aux fins d'une édition DVD... Qui plus est, les meilleurs traducteurs sont souvent reconnus comme « coauteurs » du succès d'une production : parmi les plus connus on peut nommer Elżbieta Gałązka-Salamon (elle traduit pour le cinéma et la télévision, p.ex. des séries télévisées pour enfants ou des programmes de Monty Python), Filip Łobodziński (aussi traducteur des Monty Python), Krzysztof Kowalczyk et Sylwester Misiorek (responsables, entre autres, pour le succès du Dr House), Jakub Wecsile (p.ex. traductions pour le doublage des films animés), et, le plus connu de tous, Maciej Wierzbięta, traducteur du Shrek et Astérix et Obélix. De surcroît, avec la croissance de la popularité p.ex. du Netflix, plate-forme en ligne offrant des films et séries du monde entier, et la création des studios fournissant des services de post-production, tel Deluxe Entertainment ou Sfera Studios, qui préparent aussi des traductions, le nombre de textes à traduire est devenu immense.

Pour les traductologues, un domaine encore plus intéressant sont les nouveaux médias : les films, non seulement hollywoodiens, mais beaucoup de ceux qui ne sont pas présents dans les multiplexes polonais (telle la cinématographie de Scandinavie, très riche et originelle), peuvent être vus sur Internet - pas toujours par des voies légitimes ; cela génère, à son tour, toute une « zone grise » des traductions " pirates »- des groupes d'internautes (comme celui nommé Hatak) qui préparent des traductions parfois amatrices, pleines d'erreurs, mais parfois, par contre, vraiment professionnelles et réussies ${ }^{11}$.

Comme nous l'avons déjà mentionné, la position marginale et la condition critique du cinéma polonais ne concerne pas seulement l'aspect économique - c'est juste l'une des explications possibles, et peut-être la plus importante, car c'est la raison pour laquelle notre cinématographie est toujours en retard de développement par rapport aux cinématographies plus « puissantes ». Néanmoins, il y en a d'autres. Andrzej Wajda, décédé récemment, a dit en $2006:$ «Si je savais quel type de film je dois faire, je le ferais ${ }^{12} »:$ cela peut être interprété comme un résumé d'une certaine stagnation créative qui affecte le cinéma polonais

11 Cf. A. Rębkowska, Sous-titrage dans une Europe en reconfiguration. Vers une nouvelle definition? [dans :] Vingt cinq ans après... Traduire dans une Europe en reconfiguration, éds. E. Skibińska, R. Solová, K. Gostkowska, Paris 2015, p. 273-288.

12 A. Wajda, Nie mogę stać z boku. Rozmowa Barbary Hollender z Andrzejem Wajda, « Rzeczpospolita » 2006, mars, $\mathrm{n}^{\circ} 6$. 
après 1989. On peut même conjecturer si cet « excès » de liberté, ces possibilités nouvelles d'échange avec les pays occidentaux, n'étaient pas « trop à digérer » pour nos cinéastes : à l'époque communiste le combat contre la censure, le fait d'avoir un « ennemi commun », servait d'un point de référence, forçant les artistes à un effort plus intense ; maintenant, puisque " tout est permis », et les réalisateurs et scénaristes peuvent bénéficier de l'expérience riche du monde entier, ils se contentent souvent à créer des copies carbone (pas très réussies) des films hollywoodiens. Il faut pourtant rester optimiste : depuis la chute du communisme il ne se sont écoulés que 27 ans ; peut-être la productivité et le dynamisme du cinéma américain (ainsi que d'autres cinématographies « émergentes ») commenceront-ils à inspirer les jeunes (et, sans doute, doués) cinéastes polonais. Ida de Paweł Pawlikowski qui a récemment gagné un Oscar serait peut-être un signe de renouveau dans notre cinéma?

\section{Bibliographie}

Adamczak M., Globalne Hollywood, filmowa Europa i polskie kino po 1989 roku, Gdańsk 2010.

Even-Zohar I., Miejsce literatury thmaczonej w polisystemie literackim [dans :] Współczesne teorie przekładu. Antologia, éds. P. Bukowski, M. Heydel, Kraków 2009.

Even-Zohar I., Polysystem theory revised [dans :] I. Even-Zohar, Papers in Culture Research, Tel Aviv 2010.

Kino klasyczne, éds. T. Lubelski, I. Sowińska, R. Syska, Kraków 2011.

Lubelski T., Film fabularny [dans :] T. Lubelski, Encyklopedia kultury polskiej XX wieku. Film. Kinematografia, Warszawa 1994.

Maltby R., Commercial Aesthetic [dans :] R. Maltby, Hollywood Cinema. An Introduction, Oxford 1995.

Miodek M., Polska kinematografia w zarysie, www.culture.pl (consulté le 20.11.2014).

Rębkowska A., Sous-titrage dans une Europe en reconfiguration. Vers une nouvelle definition? [dans :] Vingt cinq ans après... Traduire dans une Europe en reconfiguration, éds. E. Skibińska, R. Solová, K. Gostkowska, Paris 2015.

Venuti L., The Scandals of Translation: Towards an Ethics of Difference, London 1998.

Venuti L., Translation Strategy [dans :] L. Venuti, Routlege Encyclopaedia of Translation Studies, London 2000.

Wajda A., Nie moge stać z boku. Rozmowa Barbary Hollender z Andrzejem Wajda, « Rzeczpospolita» 2006, mars, $\mathrm{n}^{\circ} 6$.

audiowizualni.pl (consulté le 20.11.2014).

www.cnc.fr/web/en/statistics (consulté le 20.11.2014).

filmweb.pl (consulté le 20.11.2014).

kino.archiwum.org (consulté le 20.11.2014).

stopklatka.pl (consulté le 20.11.2014). 